\title{
Probing neural tissue with airy light- sheet microscopy: investigation of imaging performance at depth within turbid media
}

Jonathan Nylk, Kaley McCluskey, Sanya Aggarwal, Javier A. Tello, Kishan Dholakia

Jonathan Nylk, Kaley McCluskey, Sanya Aggarwal, Javier A. Tello, Kishan Dholakia, "Probing neural tissue with airy light-sheet microscopy: investigation of imaging performance at depth within turbid media," Proc. SPIE 10070, Three-Dimensional and Multidimensional Microscopy: Image Acquisition and Processing XXIV, 100700B (17 February 2017); doi: 10.1117/12.2251921 


\title{
Probing neural tissue with Airy light-sheet microscopy: investigation of imaging performance at depth within turbid media
}

\author{
Jonathan Nylk ${ }^{\mathrm{a}}$, Kaley McCluskey ${ }^{\mathrm{a}}$, Sanya Aggarwal ${ }^{\mathrm{b}}$, Javier A. Tello ${ }^{\mathrm{b}}$, and Kishan Dholakia ${ }^{\mathrm{a}}$ \\ ${ }^{a}$ SUPA, School of Physics and Astronomy, University of St Andrews, North Haugh, St \\ Andrews, Fife, KY16 9SS \\ ${ }^{\mathrm{b}}$ School of Medicine, University of St Andrews, North Haugh, St Andrews, Fife, KY16 9TF
}

\begin{abstract}
Light-sheet microscopy (LSM) has received great interest for fluorescent imaging applications in biomedicine as it facilitates three-dimensional visualisation of large sample volumes with high spatiotemporal resolution whilst minimising irradiation of, and photo-damage to the specimen. Despite these advantages, LSM can only visualise superficial layers of turbid tissues, such as mammalian neural tissue. Propagation-invariant light modes have played a key role in the development of high-resolution LSM techniques as they overcome the natural divergence of a Gaussian beam, enabling uniform and thin light-sheets over large distances. Most notably, Bessel and Airy beam-based light-sheet imaging modalities have been demonstrated. In the single-photon excitation regime and in lightly scattering specimens, Airy-LSM has given competitive performance with advanced Bessel-LSM techniques. Airy and Bessel beams share the property of self-healing, the ability of the beam to regenerate its transverse beam profile after propagation around an obstacle. Bessel-LSM techniques have been shown to increase the penetration-depth of the illumination into turbid specimens but this effect has been understudied in biologically relevant tissues, particularly for Airy beams. It is expected that Airy-LSM will give a similar enhancement over Gaussian-LSM. In this paper, we report on the comparison of Airy-LSM and GaussianLSM imaging modalities within cleared and non-cleared mouse brain tissue. In particular, we examine image quality versus tissue depth by quantitative spatial Fourier analysis of neural structures in virally transduced fluorescent tissue sections, showing a three-fold enhancement at $50 \mu \mathrm{m}$ depth into non-cleared tissue with AiryLSM. Complimentary analysis is performed by resolution measurements in bead-injected tissue sections.
\end{abstract}

Keywords: Light-sheet microscopy; LSM; Airy beam; Tissue imaging; turbid media; neuroscience.

\section{INTRODUCTION}

Light-sheet microscopy (LSM) has emerged as a powerful fluorescence imaging technique for biomedical research. LSM facilitates three-dimensional (3D) imaging of large sample volume with high spatial and temporal resolution and minimises irradiation of the sample. It is no wonder that LSM is ideally suited for imaging studies in developmental biology ${ }^{1}$ and neuroscience. ${ }^{2,3}$ Imaging in relatively transparent and low-scattering specimens can be achieved using existing LSM systems, ${ }^{2}$ it is desirable to be able to image in more turbid tissues such as mammalian brains. Few studies have investigated the performance of LSM in such media, and most have approached this from a theoretical perspective. ${ }^{4}$

While propagation-invariant beams, such as Bessel and Airy beams, have been shown to be beneficial for high-resolution LSM over an extended field-of-view (FOV), ${ }^{5-9}$ they additionally have the ability to reform their original transverse profile after propagation through an obstacle. This so called "self-healing" has been extensively studied for both Bessel beams ${ }^{10-12}$ and Airy beams ${ }^{12-17}$ but have only traditionally considered amplitude based obstructions. In practice, scattering in biological tissue will result from a mixture of amplitude and phase objects. Some recent studies have shown that Bessel beam based imaging modalities perform better than their Gaussian counterparts in the presence of turbid media. ${ }^{5,18}$

Further author information: (Send correspondence to J.N.)

J.N.: E-mail: jn78@st-andrews.ac.uk

Three-Dimensional and Multidimensional Microscopy: Image Acquisition and Processing XXIV, edited by

Thomas G. Brown, Carol J. Cogswell, Tony Wilson, Proc. of SPIE Vol. 10070, 100700B

(c) 2017 SPIE · CCC code: 1605-7422/17/\$18 - doi: 10.1117/12.2251921

Proc. of SPIE Vol. 10070 100700B-1

Downloaded From: https://www.spiedigitallibrary.org/conference-proceedings-of-spie on 30 Oct 2019 
In this paper, we aim to demonstrate that Airy-LSM (ALSM) offers similar improvements in image quality and imaging depth over Gaussian-LSM (GLSM) as has been observed for Bessel-LSM. We provide a quantitative analysis of images acquired by both modalities on common tissue sections from mouse brains. A windowed Fourier analysis method is developed to quantify image quality in images of fluorescently labelled neurons and homogeneous point-like resolution probes were used to quantify maximum imaging depth in tissue. Optical clearing is used to tune the scattering properties of the tissue, adding an extra degree of freedom to the study.

\section{METHODS}

This section describes the ALSM system, the preparation of neural tissues used in this study, and the windowed Fourier analysis method and spot-finding algorithm we used to quantify image quality.

\subsection{Airy light-sheet microscope}

The ALSM is described in detail elsewhere. ${ }^{8}$ Throughout this paper, the laboratory and tissue reference frame is referred to with primed coordinates, $x^{\prime}, y^{\prime}$, and $z^{\prime}$, where $z^{\prime}=0$ defines the top surface of the tissue section. The microscope reference frame is defined by unprimed coordinates, $x, y$, and $z$, where $z$ is the optic axis of the detection objective lens and light-sheet propagation is in the positive $x$ direction (see Fig. 1).

Images of tissue sections were acquired using both GLSM and ALSM (Airy parameter: $\alpha=7^{8}$ ) modalities. Both modalities were used to image the same regions of the tissue to enable direct comparison. Isotropic resolution of $800 \mathrm{~nm}$ was expected for both modalities within their high resolution FOV. The FOV for GLSM is $16 \mu \mathrm{m}$, beyond which the resolution rapidly decays. For ALSM, the FOV is $340 \mu \mathrm{m} .{ }^{8}$ Z-stacks were acquired over an axial distance of $200 \mu \mathrm{m}$ with z-plane spacing of 400nm. The illumination power was kept constant for all experiments at $240 \mu \mathrm{W}$. All ALSM datasets were deconvolved as described by Vettenburg et al. ${ }^{8}$ Deconvolution was not performed on GLSM datasets, as this is not a strict requirement for GLSM and introduces strong artefacts into regions of the image at the edge of, and outwith, the high-resolution FOV.

\subsection{Mouse breeding and husbandry}

Animal experiments were reviewed and approved by the University of St Andrews Animal Ethics and Welfare Committee under Dr Tello's Home Office Project License 70/7924. A Cre recombinase (Cre)-dependent adenoassociated virus vector was used to target expression of mCherry (a monomeric red fluorescent protein) to hypothalamic Kiss1 ${ }^{+}$neurons in Kiss1-creGFP mice. All breeding and husbandry was performed at the University of St. Andrews, St. Mary's Animal Unit. Mice heterozygous for the Kiss1-creGFP locus were obtained by

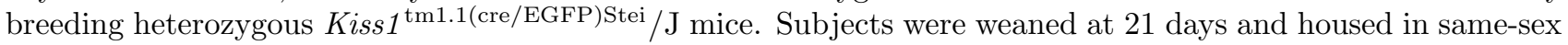
groups, under regular light-dark cycles (12h light, $12 \mathrm{~h}$ dark) with food and water available ad libitum.

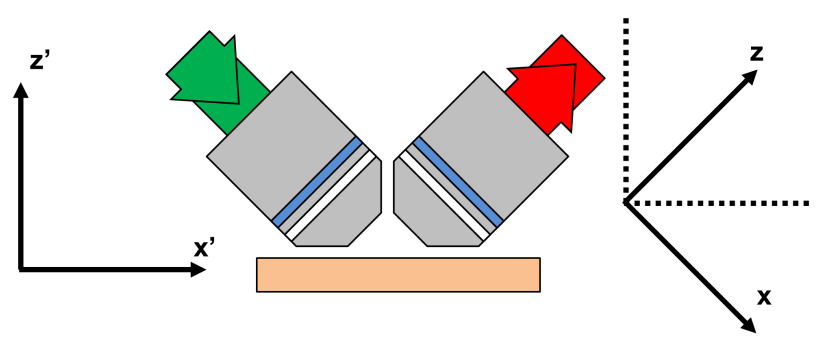

Figure 1. Schematic of LSM objective lenses oriented at $45^{\circ}$ to the $z^{\prime}$-axis (vertical) with illumination (green) along the $x$-axis and fluorescence detection (red) along the $z$-axis. Unprimed coordinates, $x$ and $z$ : microscope reference frame, primed coordinates, $x^{\prime}$ and $z^{\prime}$ : laboratory reference frame. $y=y^{\prime}$. 


\subsection{Preparation of virally transduced tissue}

Adult female heterozygous Kiss1-creGFP mice were anaesthetised with isofluorane, and viral particles (AAV; AAV1/2-Ef1a-DIO-mCherry-wPRE; $1.75 \times 10^{11} \mathrm{gc} / \mathrm{ml}$; prepared in-house as described by McClure et al ${ }^{19}$ ) were stereotaxically injected bilaterally into the hypothalamic arcurate nucleus (coordinates: AP $-1.6, \mathrm{ML} \pm 0.3, \mathrm{DV}$ -5.9 ) using a pulled glass pipette at a volume of $400 \mathrm{~nL} /$ side, at a rate of $75 \mathrm{~nL} / \mathrm{min}$ using pressure injection. After surgery, mice were returned to their cages for 3 weeks to allow for viral vector activation.

\subsection{Tissue preparation}

Animals were deeply anaesthetised with an overdose of sodium pentobarbital $(100 \mathrm{mg} / \mathrm{kg})$ and transcardially perfused with 0.1M PBS (pH 7.4) followed by 4\% PFA in PBS (pH 7.4). Brains were removed from the skull and post-fixed overnight in 4\% PFA in PBS and subsequently cryopreserved in $30 \%$ sucrose in $0.1 \mathrm{M}$ PBS. The brains were sectioned using a Compresstome vibratome (Precisionary Instruments VF-300) at a thickness of $400 \mu \mathrm{m}$.

\subsection{Preparation of bead-injected tissue}

Adult female wild type mice were anaesthetised as described above. Fluorescent beads (Duke Scientific R600, $600 \mathrm{~nm}$ diameter polystyrene, red fluorescence), diluted 1:50 in PBS, were stereotaxically injected with a volume of $500 \mathrm{~nL} /$ side bilaterally into the arcuate nucleus as described previously. Mice were culled $2 \mathrm{~h}$ following bead injection and post-fixed as described above. After clearing, the density of beads was significantly reduced and a further injection was performed on the fixed tissue.

\subsection{Optical clearing}

Tissue sections were optically cleared using TDE as described by Constantini et al. ${ }^{20}$ The cleared tissue sections were embedded in 1\% LMP agarose gel made with 47\% TDE/PBS buffer and immersed in $47 \%$ TDE/PBS during imaging. Non-cleared tissue was embedded in 1\% LMP agarose gel made with 0.1M PBS buffer and immersed in $0.1 \mathrm{M}$ PBS during imaging.

\subsection{Windowed Fourier analysis method}

The windowed Fourier analysis method used for image analysis is described below.

The spectral magnitude within the $n^{\text {th }}$ spectral window of an image of the $x^{\prime}-y^{\prime}$ plane, $S_{n}\left(z^{\prime}\right)$, is given by:

$$
S_{n}\left(z^{\prime}\right)=\frac{\int_{k_{r, n-1}}^{k_{r, n}} \int_{0}^{2 \pi}\left|\tilde{I}\left(k_{r}, k_{\theta} ; z^{\prime}\right)\right| k_{r} d k_{r} d k_{\theta}}{\int_{k_{r, n-1}}^{k_{r, n}} \int_{0}^{2 \pi} k_{r} d k_{r} d k_{\theta}}
$$

where $\tilde{I}\left(k_{r}, k_{\theta} ; z^{\prime}\right)$ is the Fourier transform of the image plane $I\left(x^{\prime}, y^{\prime} ; z^{\prime}\right)$ in cylindrical coordinates and $k_{r, n}$ is the radial spatial frequency separating spectral windows in $10 \%$ increments of the diffraction limit:

$$
k_{r, n}=\frac{n}{10} \frac{2 \mathrm{NA}}{\lambda}
$$

The enhancement factor, describing relative imaging performance, within a given spectral window is then given by the ratio between Airy and Gaussian imaging modalities:

$$
E F_{n}\left(z^{\prime}\right)=\frac{S_{n}\left(z^{\prime}\right)_{\text {Airy }}}{S_{n}\left(z^{\prime}\right)_{\text {Gaussian }}}
$$

\subsection{Spot-finding algorithm and FWHM measurements}

A spot-finding algorithm written in-house in MATLAB was used to locate individual point-spread-functions (PSFs) within the size range $0.6-4.0 \mu \mathrm{m}$ and fit one-dimensional (1D) Gaussian functions along the lateral $(x, y)$ and axial $(z)$ directions. The full-width-half-maximum (FWHM) was then determined from the fitted Gaussian profile. 


\section{RESULTS AND DISCUSSION}

The following section describes the experimental results of our study. Volumetric image stacks acquired by GLSM and ALSM in non-cleared and cleared tissue were analysed. Virally transduced tissue is analysed using a windowed Fourier method, and FWHM analysis is performed on bead-injected tissue .

\subsection{Fourier analysis of virally transduced fluorescent tissue sections}

The use of Fourier analysis for analysis of image quality in thick tissues is motivated by the large variability of biological structures and the different features observed at different length scales. Our Fourier analysis method is described in Section 2.7 and we investigated this method as a function of tissue depth $z^{\prime}$ in order to concisely capture the effect of aberrations on both the illumination and detection pathways with a single metric. ${ }^{4}$ Due to the large difference in FOV between the imaging methods ( $16 \mu \mathrm{m}$ for GLSM, $340 \mu \mathrm{m}$ for ALSM) the images were apodized with a Gaussian function along the $x$-axis with a half-width of $8 \mu \mathrm{m}$ and centred of the focus of the Gaussian light-sheet in order to compare only the regions where similar resolution should be achieved (see Fig. 2). Taking the average of $E F_{n}\left(z^{\prime}\right)$ over the high-frequency spectral bands $(60-100 \%$ of $2 \mathrm{NA} / \lambda)$ gives an average enhancement factor, $E F_{H F}\left(z^{\prime}\right)$ that serves as a good metric for resolution as a function of tissue depth.

This analysis was performed on volumetric image stacks acquired around the surface of non-cleared and cleared tissue sections with both ALSM and GLSM. Table 1 shows the average linear fit parameters (mean \pm standard deviation) to $E F_{H F}\left(z^{\prime}\right)$ over 11 volumes from various sample regions in non-cleared tissue and over 9 volumes in cleared tissue.

The positive gradient of these fits indicate that the relative image quality of ALSM compared to GLSM increases with depth. The image quality of both modalities is expected to decrease with depth as aberrations increase, but our results show that the image quality of ALSM decreases at a slower rate than for GLSM. In cleared tissue, the degree of sample-induced aberrations is greatly reduced and it can be expected that the relative improvement would be less. This is mirrored in our results as the gradient of $E F_{H F}\left(z^{\prime}\right)$ in cleared tissue is 5 times lower than in non-cleared tissue.
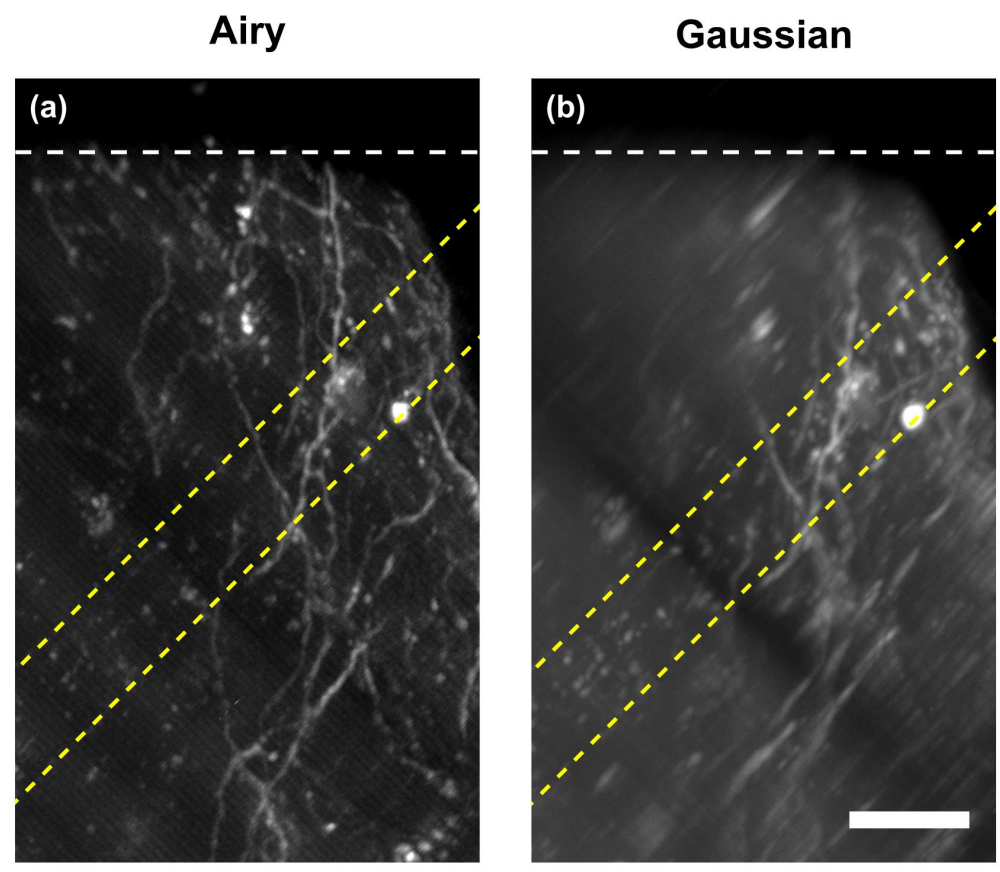

Figure 2. Maximum intensity projections $\left(x^{\prime}-z^{\prime}\right.$ view) of virally transduced tissue sections imaged by ALSM (a) and GLSM (b). Tissue surface $\left(z^{\prime}=0\right)$ is indicated by a dashed white line. Width of Gaussian apodization around Gaussian focus is indicated by yellow dashed lines. Scale bar: $50 \mu \mathrm{m}$. 
Table 1. Average linear fit parameters for $E F_{H F}\left(z^{\prime}\right)$ in non-cleared and cleared tissue sections.

\begin{tabular}{|l|c|c|c|}
\hline Tissue Type & Sample Size & $E F_{H F}\left(z^{\prime}\right)$ gradient $\left(\mu \mathrm{m}^{-1}\right)$ & $E F_{H F}\left(z^{\prime}\right)$ y-intercept \\
\hline Non-cleared & 11 & $0.04 \pm 0.02$ & $1.2 \pm 0.2$ \\
\hline Cleared & 9 & $0.008 \pm 0.007$ & $1.6 \pm 0.4$ \\
\hline
\end{tabular}

If no aberrations are present, both beam types would be expected to give equal performance $\left(E F_{H F}\left(z^{\prime}\right)=1\right)$ and so the y-intercept is expected to be unity. In both tissue types, the y-intercept is greater than unity. In non-cleared tissue this is attributed to a small refractive index mismatch between the agarose the sample is embedded in and the immersion medium (PBS). The y-intercept is higher still in cleared tissue which we attribute to stronger spherical aberration due to the refractive index mismatch between the objective lens and the immersion medium for cleared samples (TDE; $n=1.42 \pm 0.01^{20}$ ). Additional control experiments imaging isolated sub-diffraction limited beads showed a reduction in resolution of approximately $20-30 \%$ when imaging in the TDE buffer.

\subsection{Analysis of homogeneous features in bead-injected tissue}

Measurements in bead-injected tissue were also used to control for the variability of feature size encountered in the virally transduced tissue. The tissue was cut through the site and positioned in the microscope such that the beads were located at the tissue edge closest to the detection objective lens and volumetric images were acquired in cleared tissue with ALSM and GLSM and FWHM measurements of bead dimensions were determined (see Section 2.8). Figure 3 shows maximum intensity projections $\left(x^{\prime}-z^{\prime}\right.$ view) composite of 3 image stacks acquired with GLSM and ALSM at different tissue depths. Table 2 shows linear fits of the FWHM measurements versus tissue depth, $z^{\prime}$ for this data. Sample size indicates the number of individual beads identified by the spot-finding algorithm.

Table 2 shows very similar results for the lateral resolution of each beam type, this is expected as the beam type is expected to influence only the axial resolution. Unexpectedly, for the axial resolution, the gradient is higher for ALSM, indicating more rapidly degrading resolution than for GLSM and in contrary to our results in Section 3.1. Visually, from Fig. 3 it appears that the resolution degrades more rapidly for GLSM than ALSM. This discrepancy can be attributed to the spot-finding algorithm which searches for isolated PSFs ranging between $0.6-4.0 \mu \mathrm{m}$. In the Gaussian image, the axial resolution is highly spatially-variant, outwith the high resolution FOV defined in Section 2.1 the axial resolution quickly degrades and can be in excess of $16 \mu \mathrm{m}$. When the axial PSF becomes larger, the likelihood of multiple PSFs overlapping also greatly increases meaning that even when individual PSFs are still within the upper limit of the algorithm, they may overlap and their combined size will be rejected by the algorithm. In this way, the algorithm filters out low resolution features in the GLSM data and therefore underestimates the size of PSFs found in this dataset.

Additionally, the deepest feature to be identified by the spot finding algorithm was found at a depth of

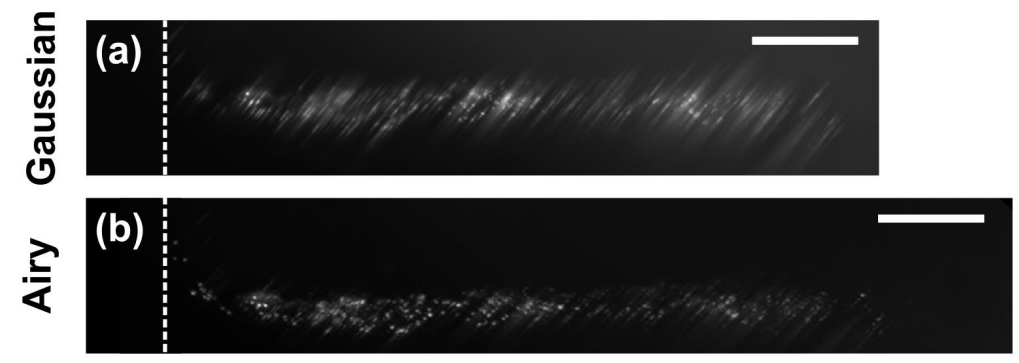

Figure 3. Maximum intensity projections $\left(z^{\prime}-x^{\prime}\right.$ view) of bead-injected tissue sections imaged by GLSM (a) and ALSM (b). Tissue surface $\left(z^{\prime}=0\right)$ is indicated by a dashed white line. Scale bar: $50 \mu \mathrm{m}$. 
Table 2. Average linear fit parameters for $\operatorname{FWHM}\left(z^{\prime}\right)$ in cleared tissue sections

\begin{tabular}{|c|c|c|c|c|}
\hline Beam Type & Sample Size & Fit Orientation & FWHM $\left(z^{\prime}\right)$ gradient & FWHM $\left(z^{\prime}\right)$ y-intercept $(\mu \mathrm{m})$ \\
\hline Gaussian & 86 & $\begin{array}{l}\text { Lateral } \\
\text { Axial }\end{array}$ & $\begin{array}{l}(1.9 \pm 0.6) \times 10^{-3} \\
(2.2 \pm 0.6) \times 10^{-3}\end{array}$ & $\begin{array}{c}1.1 \pm 0.1 \\
1.16 \pm 0.09\end{array}$ \\
\hline Airy & 257 & $\begin{array}{l}\text { Lateral } \\
\text { Axial }\end{array}$ & $\begin{array}{l}(1.7 \pm 0.2) \times 10^{-3} \\
(2.6 \pm 0.3) \times 10^{-3}\end{array}$ & $\begin{array}{l}1.18 \pm 0.04 \\
1.44 \pm 0.06\end{array}$ \\
\hline
\end{tabular}

$251 \pm 1 \mu \mathrm{m}$ in the GLSM data, but at $330 \pm 1 \mu \mathrm{m}$ in the ALSM data, further suggesting that ALSM can increase the penetration depth of the light-sheet into tissue.

\section{SUMMARY AND CONCLUSIONS}

We have used two analysis methods and two types of neural tissues with different degrees of sample-induced aberrations to investigate the effect of illumination beam type on imaging performance in LSM. Gaussian and Airy light-sheets were compared. We developed an image quality metric around the magnitude of the spatial Fourier transform of an image within a certain frequency range. This method can more generally be applied across a wide range of image modalities when comparisons on a common sample are possible. The enhancement factor followed a relatively linear trend and indicated an ALSM image retains approximately 3.2 times more high frequency content than GLSM at $50 \mu \mathrm{m}$ depth within mouse brain tissue. In cleared tissue, the aberrations were significantly lower, and the gradient of the enhancement factor was also lower. Finally, using homogeneous point-like resolution probes the maximum imaging depth with each modality was tested. Both ALSM and GLSM gave similar resolution estimates based on FWHM measurements but small features could be observed at $30 \%$ greater tissue depth with ALSM.

Our analysis shows that the use of an Airy beam for light-sheet illumination does offer advantages in terms of image quality and imaging depth which we attribute to the natural aberration resistance of the Airy beam. Given that ALSM can be implemented in a compact and inexpensive manner ${ }^{9}$ it may be particularly suitable for biomedical end-users in neuroscience.

\section{ACKNOWLEDGMENTS}

This work was supported by awards to KD from the UK Engineering and Physical Sciences Research Council under grant EP/J01771X/1, the 'BRAINS' 600th anniversary appeal, and Dr. E. Killick. We would also like to thank The Northwood Trust and The RS Macdonald Charitable Trust for funding support (award to JAT). KD acknowledges support of a Royal Society Leverhulme Trust Senior Fellowship.

\section{REFERENCES}

[1] Huisken, J., Swoger, J., Del Bene, F., Wittbrodt, J., and Stelzer, E. H. K., "Optical sectioning deep inside live embryos by selective plane illumination microscopy.," Science (New York, N.Y.) 305, 1007-9 (aug 2004).

[2] Ahrens, M. B., Orger, M. B., Robson, D. N., Li, J. M., and Keller, P. J., "Whole-brain functional imaging at cellular resolution using light-sheet microscopy.," Nature methods 10, 413-20 (may 2013).

[3] Yang, Z., Haslehurst, P., Scott, S., Emptage, N., and Dholakia, K., "A compact light-sheet microscope for the study of the mammalian central nervous system," Scientific Reports 6, 26317 (may 2016).

[4] Glaser, A. K., Wang, Y., and Liu, J. T., "Assessing the imaging performance of light sheet microscopies in highly scattering tissues," Biomedical Optics Express 7, 454 (jan 2016).

[5] Fahrbach, F. O., Simon, P., and Rohrbach, A., "Microscopy with self-reconstructing beams," Nature Photonics 4, 780-785 (sep 2010). 
[6] Planchon, T. A., Gao, L., Milkie, D. E., Davidson, M. W., Galbraith, J. A., Galbraith, C. G., and Betzig, E., "Rapid three-dimensional isotropic imaging of living cells using Bessel beam plane illumination," Nature Methods 8(5), 417-423 (2011).

[7] Olarte, O. E., Licea-Rodriguez, J., Palero, J. A., Gualda, E. J., Artigas, D., Mayer, J., Swoger, J., Sharpe, J., Rocha-Mendoza, I., Rangel-Rojo, R., and Loza-Alvarez, P., "Image formation by linear and nonlinear digital scanned light-sheet fluorescence microscopy with Gaussian and Bessel beam profiles.," Biomedical optics express 3, 1492-505 (jul 2012).

[8] Vettenburg, T., Dalgarno, H. I. C., Nylk, J., Coll-Lladó, C., Ferrier, D. E. K., Čižmár, T., Gunn-Moore, F. J., and Dholakia, K., "Light-sheet microscopy using an Airy beam.," Nature methods 11(5), 541-4 (2014).

[9] Yang, Z., Prokopas, M., Nylk, J., Coll-Lladó, C., Gunn-Moore, F. J., Ferrier, D. E. K., Vettenburg, T., and Dholakia, K., "A compact Airy beam light sheet microscope with a tilted cylindrical lens.," Biomedical optics express 5, 3434-42 (oct 2014).

[10] Bouchal, Z., Wagner, J., and Chlup, M., "Self-reconstruction of a distorted nondiffracting beam," Optics Communications 151, 207-211 (jun 1998).

[11] Chen, Y. and Liu, J. T., "Characterizing the beam steering and distortion of Gaussian and Bessel beams focused in tissues with microscopic heterogeneities," Biomedical Optics Express 6, 1318-1330 (apr 2015).

[12] Mazilu, M., Stevenson, D., Gunn-Moore, F., and Dholakia, K., "Light beats the spread: non-diffracting beams," Laser 83 Photonics Reviews 4, 529-547 (sep 2010).

[13] Broky, J., Siviloglou, G. A., Dogariu, A., and Christodoulides, D. N., "Self-healing properties of optical Airy beams," Optics Express 16, 12880 (aug 2008).

[14] Baumgartl, J., Mazilu, M., and Dholakia, K., "Optically mediated particle clearing using Airy wavepackets," Nature Photonics 2, 675-678 (sep 2008).

[15] Chu, X., Zhou, G., and Chen, R., "Analytical study of the self-healing property of Airy beams," Physical Review A 85, 013815 (jan 2012).

[16] Qian, Y. and Wyrowski, F., "Evolution of self-healing characteristic on optical Airy beam," Optik 125(15), 3876-3879 (2014).

[17] Zhang, L., Ye, F., Cao, M., Wei, D., Zhang, P., Gao, H., and Li, F., "Investigating the self-healing property of an optical Airy beam," Optics Letters 40, 5066 (nov 2015).

[18] Fahrbach, F. O., Gurchenkov, V., Alessandri, K., Nassoy, P., and Rohrbach, A., "Self-reconstructing sectioned Bessel beams offer submicron optical sectioning for large fields of view in light-sheet microscopy," Optics Express 21, 11425 (may 2013).

[19] McClure, C., Cole, K. L. H., Wulff, P., Klugmann, M., and Murray, A. J., "Production and Titering of Recombinant Adeno-associated Viral Vectors," Journal of Visualized Experiments, e3348 (nov 2011).

[20] Costantini, I., Ghobril, J.-P., Di Giovanna, A. P., Allegra Mascaro, A. L., Silvestri, L., Müllenbroich, M. C., Onofri, L., Conti, V., Vanzi, F., Sacconi, L., Guerrini, R., Markram, H., Iannello, G., and Pavone, F. S., "A versatile clearing agent for multi-modal brain imaging.," Scientific reports 5, 9808 (jan 2015). 